\title{
Parents of Psychiatrically Hospitalized Children: A Decade of Changing Perceptions
}

\section{Ann Jacobs Mooney, A.C.S.W.}

ABSTRACT: Psychiatric hospitals for children have changed dramatically during the last decade. The lengths of hospitalizations have been shortened; the psychopathology of children qualifying for admission is more severe, often with neurological or biochemical components. In some hospitals, there has been an increasing emphasis on research. All of these changes have affected the staff's perceptions of the children's parents; these changes appear to have resulted in a more supportive, less critical attitude toward these parents. This may be significant in increasing parents' confidence in coping with their child's illness and their family's stress. The need for empirical, longitudina! research is emphasized.

When a child is hospitalized on a psychiatric unit, the parents are crucial to the evaluation and treatment of that child. While the importance of parents to their child's inpatient treatment may seem obvious, it is a topic which has been surprisingly rare in recent literature, and certainly warrants further empirical study (Blotcky et al., 1984). During the last decade, I have been a social worker whose roles have included child therapist, parent and family therapist, and research co-investigator at a children's psychiatric hospital affiliated with a large midwestern university. I have found that the view of these children's parents, in the eyes of hospital staff, has been a slowly evolving one. A review of the evolution of these perceptions may have significance both for clinical implications and for potential research.

\footnotetext{
Ms. Mooney is a lecturer at the University of Michigan's School of Social Work. She is also a senior social worker at the Psychological Clinic, also affiliated with the University of Michigan. Address correspondence to Ms. Mooney at 2106 Arborview, Ann Arbor, Michigan 48103.

The research mentioned in this article was funded by the American Cancer Society. 


\section{Changing Perceptions of Parents}

There have been many changes in program structures, treatment approaches, and philosophy, which have paralleled these changing views of patients' parents. In the late 1970's, I worked on inpatient psychiatric units which hospitalized children for an average length of stay of at least one year, with a range of treatment time from six months up to three years. The children were three to thirteen years of age. When they were initially admitted, they often saw their parents once per week; that contact occurred on the ward and was supervised by nursing staff. The next stage was one in which visits were on the ward but unsupervised; later the parents could take the child for a pass on hospital grounds. Eventually, most children would be granted passes beyond the hospital's limits. Prior to discharge many parents would be allowed to take their children home on overnight passes. This process of loosening supervisory control over parent-child contact usually took many months, and sometimes a year or more. Meanwhile, parents were seen by a unit social worker once per week for parent guidance sessions.

At that time the mode of treatment for the inpatient children was usually a psychoanalytic, individually oriented approach focused on the child's internal conflicts. Because of the intense long-term relationships between staff and the patients, a strong identification with, and protectiveness of, these children often developed. Clinical meetings frequently included discussions of concerns about countertransference responses to the parents. Social workers were usually the only staff working directly and regularly with the parents, and often found themselves identified negatively with the parents. Other staff would at times become frustrated with the parents' therapist, claiming that if the social worker could induce change in the parents, the child's difficulties would be alleviated to a great extent.

Indeed, even the social workers, along with many other staff, often viewed the parents as pathogenic agents who were solely responsible for disrupting what would have been the normal psychological development of their children. Phrases emerged such as "parentectomy"; at times it was simply felt that the recommended treatment approach that could produce the best results for the child was the removal of the parent from the child's life. More commonly discussed was the treatment goal of "inoculating the child against the parents", or helping the child to continue on a healthier developmental path by making him or her more immune to the influence of the parent. Over- 
all, it was clear that the parents often were seen as disturbed individuals themselves. In some cases, this was clearly warranted by parents' actual characterological problems. However, in retrospect, overidentification with the inpatient children in many cases may have resulted in the assumption of parental psychopathology without adeqate substantiation of such diagnoses.

In the early 1980's, one of the inpatient children's wards became a short-term diagnostic unit, focusing on brief assessments and interventions with possible research components. Due to both changes in philosophy as well as pressure from third-party-payment organizations, the length of stay became dramatically shorter, averaging approximately six weeks. Parents are encouraged to visit their hospitalized children more often, and more frequent participation in parent guidance and family therapy sessions is expected. Generally, all hospital-based interventions are more intensive; however, passes for the children off hospital grounds are quite rare.

Thus, the long-term relationships that existed between children and staff over a period of many months or years, are no longer a component of the program. There has been a lessening of the intense identification with the children, as well as a decrease in the countertransference reactions to these children's parents. Also there is a much stronger family focus in the treatment of these children; while the parent sometimes still is seen as a disturbed or disturbing factor in a "sick family", there is not the strong negative response to parents which was seen in the more intense, individually focused, long-term treatment milieu.

Finally, due to the constraints of new policies for many insurance companies, the types of children who are now being considered appropriate for inpatient admissions are more often those with neurological or biochemical components to their psychiatric illnesses. With such organic difficulties evident in the child, it appears to have become easier for the staff to empathize with the parents in regard to the often overwhelming task of parenting a child with serious developmental problems. More often there is definite evidence that helps to account for a child's behavioral difficulties: a CAT scan identifies the site of neurological involvement, or a response to pharmacotherapy dramatically reduces a child's hyperactivity. Paralleling this increase in medical substantiation of neurological or biochemical factors in the children's psychopathology, has been a more supportive, less critical attitude toward their parents.

There was a similar attitude apparent in the literature regarding 
parents of institutionalized mentally retarded children in the 1960's, prior to current deinstitutionalization trends (Andrew et al., 1965; Downey, 1965; Klaber, 1968; Skelton, 1972). The tone of these articles was often quite sympathetic, focusing on topics such as stresses on parents due to the care required for the retarded child, or concern for parents torn by attempts to divide their time between the retarded child's needs and those of the normal siblings (Downey, 1965; Skelton, 1972).

This focus is noticeably different than that of many more current articles about parents of children hospitalized because of severe behavioral disturbances without a verifiable neurological, biochemical, or intellectual deficit. These articles are more likely to focus on specific parental interactions with the child, and parental pathology or diagnosis (Dalton, 1987; Winsberg, 1980). Again, there are clearly cases for which such a focus is quite salient to the evaluation and treatment of a child. Still, it is noteworthy that none of the articles on parents of mentally retarded children mentioned assessing parents' mental health; also, none of the articles about parents of psychiatrically disturbed children addressed concerns about stress on parents due to driving distances and time involved in hospital visitation or other such concrete yet important demands on the parent of any hospitalized or institutionalized child. In general, the tone was consistently more critical and less supportive of parents of psychiatrically ill children, when compared with parents of children with more obvious or verifiable intellectual or psychological limitations. This dynamic certainly was one factor in lessening the negative countertransference of our hospital staff, as more children were admitted with neurological or biochemical complications.

\section{Studying the Parents}

More recently, my role with these parents changed again, from that of a social worker doing assessments and therapy with short-term diagnostic cases, to that of a co-investigator on two different research projects involving subjects from the same unit. This experience further broadened my perception of the role of parents during their children's hospitalizations.

The research project which I have used primarily for these observations is a small pilot study funded by the American Cancer Society which involves the families of children with chronic illnesses. We are investigating the impact of family functioning on the psychosocial de- 
velopment of children with asthma, cancer, and psychiatric disorders. The psychiatric population included children whose diagnoses we considered appropriate for inclusion in the behavioral disorder category, e.g., attention deficit disorder with hyperactivity, oppositional disorder, conduct disorder, adjustment disorder, major depressive disorder, intermittent explosive disorder, separation anxiety disorder, and pervasive developmental disorder.

My varying role with the parents as a co-investigator has been extremely interesting. In addition to continuing to develop my own perceptions of the parents' role, I was able to collect information on the perceptions of the other co-investigators when they interviewed parents of children from the psychiatric population. All other persons interviewing the parents were also social workers; however, some had had experience only with the asthma or cancer populations prior to this study.

Other co-investigators stated that they had expected the parents of the psychiatric population to appear to have more psychological difficulties than parents of the other two populations; however, these social workers found that most of these parents were cooperative, articulate individuals whose general presentations did not vary significantly from parents of the other two populations.

The major difference noted between these groups was that parents of the children with asthma or cancer were very eager to talk about the health of their children, i.e., what the child could still accomplish in spite of his or her illness. However, parents of the children with psychiatric or behavioral disorders were more consistently eager to talk about the child's problems and rarely seemed to discuss the child's accomplishments and strengths, or positive aspects of the child's mental health. Thus, while the parents of the cancer and asthma groups were reticent to discuss the chronic illness, the parents of the psychiatric population seemed clearly relieved to discuss their children's illness.

There are several hypotheses as to why this interesting distinction emerged during the interviewing and data collection of this research. In the past it might have been assumed that the parents of the children with psychiatric problems were invested in their child's illness in an unhealthy way and perhaps received gratification from the child's behavioral acting out; thus the parent would be more apt to focus on the problems in an interview or questionnaire. Another assumption could be that throughout the child's life, the parents had focused primarily on the negative aspects of the child's functioning; 
such a focus could have impacted negatively on the child's development, particularly in the area of self-esteem. Therefore the parent's perception of the child may have exacerbated whatever problems arose in the child's psychological and behavioral development. Or perhaps one could hypothesize that the parents of the children with cancer and asthma were defensively, and possibly adaptively, denying elements of their child's chronic illness.

\section{The Social Stigma}

However, I believe more accurate inferences can be made from these observations. When a child develops asthma or cancer, it is a clear biological condition for which parents usually receive sympathy and support from relatives, friends, or social organizations, to help them cope with the stress of their child's illness. But for the parents of children with psychiatric disturbances, there is often a social stigma which leaves the parents uncomfortable and unable to discuss their stresses, frustrations, or fears. Many times when they do discuss their child's behavior with others, the response they receive involves blame, and criticism for poor parenting. For example, there is a great lack of public understanding of biochemical problems such as hyperkinesis; for the majority of parents of hyperactive children, there are continual battles with school staff, friends, and relatives who blame the parents for "not adequately disciplining and controlling" the child. When these children are not appropriately assessed and diagnosed, parents understandably struggle with feelings of guilt, confusion, anger, and ultimately helplessness. Our research indicated that, for many of these families, social isolation is more common than for families in which a child has cancer or asthma.

For the parents of children with behavioral disorders, it therefore makes sense as to why, in a setting focused on psychiatric problems, there would be great relief in finally discussing the tremendous difficulties and helplessness which they had been experiencing, often for years. Many of these parents stated that they did indeed worry that their own shortcomings had somehow caused the child's problems. Often parents expressed hope that with an adequate evaluation during the hospitalization, some issues might be clarified; they hoped that a more specific diagnosis might lead to a more workable plan for treatment.

Another interesting observation resulting from the research project 
was that the parents of the psychiatric population were much more willing to participate in this project than the parents of the children with asthma or cancer. There was a ten percent refusal rate for the parents of the psychiatric population, while approximately thirty percent of the other groups' parents refused participation.

\section{Helping Helps}

When parents were asked to participate in this research protocol, they were informed that this research was not going to affect or help either them or their child; however, we explained that our goal was for the ultimate results of the project to help the families of other children with problems similar to those of their child. Many of the parents of the psychiatric population commented that if they could help any other parents avoid the painful problems they had encountered, they would be eager to offer their time. Many discussed their feelings that for years they were continually having to ask for the help of others. None described having had any opportunities to be in a position to do anything helpful for others in situations like theirs.

Thus, one unanticipated observation resulting from our research was that in appealing to the healthier, altruistic motivations of the parents of the psychiatric population, they became more aware of their own strengths and emotional health. There was a noticeable increase in pride resulting from the generosity of having donated their time; many stated that "it felt good to be helpful" or they hoped somehow they had helped someone else. It clearly warrants further investigation to explore the impact of voluntary research participation on the self-esteem of parents of children with behavioral disorders and other psychiatric illnesses. One possible result could be the stimulation of interest in joining support groups for parents of children with psychiatric illness. But even if there is no action resulting from the parents' research participation, there is a benefit in having the hospital staff involved with these parents in a type of mutual endeavor, that is, a mutual wish to help other parents who are experiencing similar stresses and difficulties as they are. Also, the increase in pride or self-esteem which can result from research participation could be an element which contributes to the general confidence of these parents as they deal with the emotional stress on their entire family. Perhaps in some small way, it may even increase their confidence in their own abilities to cope with their child's chronic illness. 


\section{Conclusions}

During the last decade, the psychiatric hospitalization of children has changed in many ways. The length of stay in most institutions has been dramatically shortened; the psychopathology of the children admitted has become more severe, often with neurological and/or biochemical components. Many aspects of these important changes are being investigated, particularly in regard to the effectivity of shortterm treatment, and the implications of increased use of pharmacotherapy with children. But one area which has not been adequately investigated is the involvement of hospital staff with the parents of inpatient children. While there has been an occasional reporting of a program which attempts to address this concern (Birmingham et al., 1977), there is an urgent need for empirical, longitudinal research of this important topic. Having observed the evolving nature of hospital staff's perceptions of parents of inpatient children over the last decade, one can hypothesize that the more supportive, less critical tone of the staff toward the parent may have a positive impact on the parent's self-image. A reasonable corollary to such a hypothesis would be that the resulting increase in self-esteem and confidence may strongly influence a parent's involvement with the patient's evaluation and treatment, and may positively impact on his or her ultimate ability to parent a difficult child. Thus the significance of the need for empirical study of staff-parent interaction becomes clear when one considers its potential clinical value, not only to the parent, but to the disturbed child and other family members as well.

\section{References}

Andrew, G., Kime, W., Stehman, V., \& Jaslow, R. (1965). Parental contacts along the route to institutional commitment of retarded children. American Journal of Mental Retardation, 70(3), 399-407.

Birmingham, V., Rogers, M., \& Schmidt, P. (1977). Implementing a parent involvement program in a state hospital and school for children. Children Today, 6(4), 20-23.

Blotcky, M., Dimperio, T., \& Gossett, J. 91984). Follow-up of children in psychiatric hospitals: a review of studies. American Journal of Psychiatry, 141(12), 1499-1507.

Dalton, R., Forman, M., Daul, G., \& Bolding, D. (1987). Psychiatric hospitalization of preschool children: admission factors and discharge implications. Journal of the American Academy of Child and Adolescent Psychiatry, 26(3), 308-312.

Downey, K. (1965). Parents' reasons for institutionalizing severely mentally retarded children. Journal of Health and Social Behavior, 6(3), 147-155. 
Klaber, M. (1968). Parental visits to institutionalized children. Mental Retardation, $6(6), 39-41$.

Skelton, M. (1972). Areas of parental concern about retarded children. Mental Retardation, 10(1), 38-41.

Winsberg, B., Bialer, I., Kupietz, S., Botti, E., \& Balka, E. (1980). Home versus hospital care of children with behavior disorders: 2. A controlled investigation. Archives of General Psychiatry 37(4), 413-418. 